\title{
Incidence of "never events" among weekend admissions versus weekday admissions to US hospitals: national analysis
}

\author{
Frank J Attenello, ${ }^{1}$ Timothy Wen, ${ }^{2}$ Steven Y Cen, ${ }^{3,4}$ Alvin Ng, ${ }^{5}$ May Kim-Tenser, ${ }^{3}$ Nerses Sanossian, ${ }^{3}$ \\ Arun P Amar, ${ }^{1}$ William J Mack ${ }^{1}$
}

'Department of Neurosurgery, Keck School of Medicine,

University of Southern

California, Los Angeles,

CA 90033, USA

${ }^{2}$ Keck School of Medicine,

University of Southern

California, USA

${ }^{3}$ Department of Neurology, Keck

School of Medicine, University

of Southern California, USA

${ }^{4}$ Department of Radiology, Keck

School of Medicine, University

of Southern California, USA

${ }^{5}$ Department of Preventive

Medicine, Keck School of

Medicine, University of

Southern California, USA

Correspondence to: TWen

wentimot@usc.edu

Additional material is published online only. To view please visit

the journal online (http://dx.doi. org/10.1136/BMJ.h1460)

Cite this as: $B M / 2015 ; 350: h 1460$

doi:10.1136/bmj.h1460

Accepted: 17 February 2015

\section{ABSTRACT}

OBJECTIVE

To evaluate the association between weekend admission to hospital and 14 hospital acquired conditions recently considered by the Centers for Medicare and Medicaid as "never events," serious, costly, and preventable medical errors, for which resulting healthcare costs are not reimbursed.

\section{DESIGN}

National analysis.

SETTING

US Nationwide Inpatient Sample discharge database.

PARTICIPANTS

351 million patients discharged from US hospitals, 2002-10.

\section{MAIN OUTCOME MEASURES}

Univariate rates and multivariable likelihood of hospital acquired conditions among patients admitted on weekdays versus weekends, as well as the impacts of these events on prolonged length of stay and total inpatient charges.

\section{RESULTS}

From 2002 to 2010, 351170803 patients were admitted to hospital, with $19 \%$ admitted on a weekend. Hospital acquired conditions occurred at an overall frequency of $4.1 \%$ (5.7\% among weekend admissions versus 3.7\% among weekday admissions). Adjusting for patient and hospital cofactors the probability of having one or more hospital acquired conditions was more than $20 \%$ higher in weekend admissions compared with weekday admissions (odds ratio 1.25, 95\% confidence interval 1.24 to $1.26, \mathrm{P}<0.01)$. Hospital acquired conditions have a negative impact on both hospital charges and length of stay. At least one hospital acquired condition was associated with an $83 \%(1.83,1.77$ to $1.90, \mathrm{P}<0.01)$ likelihood of increased charges and $38 \%$ likelihood of prolonged length of stay $(1.38,1.36$ to $1.41, \mathrm{P}<0.01)$.

CONCLUSION

Weekend admission to hospital is associated with an increased likelihood of hospital acquired condition, cost, and length of stay. Future protocols and staffing

\section{WHAT IS ALREADY KNOWN ON THIS TOPIC}

Multiple studies have correlated increased mortality with weekend admission

A set of "never events," termed hospital acquired conditions (HACs), have been

defined as quality measures for which resulting healthcare costs are not

reimbursed

\section{WHAT THIS STUDY ADDS}

Weekend admission was associated with an increased likelihood of HAC occurrence when adjusting for disease severity and admission urgency in multivariate analysis regulations must be tailored to the requirements of this high risk subgroup.

\section{Introduction}

"Never events" are an assembly of purportedly egregious and preventable hospital occurrences first introduced by the National Quality Forum in 2001. The list was generated in response to a heightened awareness of avertable errors leading to in-hospital morbidity and mortality. ${ }^{1}$ Defined as serious, costly, and preventable medical errors, these events were subsequently consolidated by the Centers for Medicaid and Medicare Services (CMS) into a list of hospital acquired conditions that would not qualify for federal compensation for any subsequent treatment relating to the condition (see supplementary table). ${ }^{2}$ The creation of these events is designed to shift the burden of complications from the patient and government to the individual hospitals and to motivate hospitals to accelerate patient safety programs. ${ }^{2}$ However, these events have been met with considerable resistance, with many leaders of American healthcare systems suggesting that not all hospital acquired conditions are preventable. ${ }^{2-4}$ Others suggest that CMS's policy for hospital acquired conditions could potentially drive hospitals to be less likely to treat sicker patients. ${ }^{5}$ However, a press release in December 2014 highlights the correlation between saving 50000 lives and \$12 bn ( $€ 8$ bn; $€ 11$ bn) from 2010 to 2014 , with a $17 \%$ decrease in hospital acquired conditions over that period. ${ }^{6}$ Hospital acquired conditions have been aptly increasing as a metric for quality of patient healthcare.

Previous investigations have tackled risk factors associated with inpatient complications. ${ }^{12-12}$ Though studies have attempted to isolate exposures associated with hospital acquired conditions and quantify resultant increases in attributable costs, published reports are restricted to specific medical or surgical subspecialties or patient demographics. ${ }^{127-1013}$ The occurrence of hospital acquired conditions has not been evaluated across all patient subgroups on a national scale.

Hospital acquired conditions are events thought to be preventable through effective hospital systems and administrative processes. However, healthcare delivery may be most susceptible to the occurrence of these conditions at times when staffing or standard leadership is reduced. Total numbers of hospital staff and available expertise are often diminished on weekends, ${ }^{14}$ with differences in mortality between weekend and weekday admissions documented for conditions that require aggressive intervention. Weekend admission is associated with increased mortality among all hospital admissions and within subgroups of patients with pulmonary 
embolism, stroke, subarachnoid hemorrhage, intracerebral hemorrhage, and myocardial infarction..$^{15-22}$

We established standards for rates of hospital acquired conditions across an entire inpatient sample of greater than 350 million admissions. Incidence rates are determined for weekend versus weekday admissions across all patients included in the Nationwide Inpatient Sample hospital discharge database from 2002 to 2010 . We hypothesized that weekend admission would be associated with a higher frequency of hospital acquired conditions.

\section{Methods}

Data source

We carried out a cross sectional analysis study using data from the 2002 to 2010 extracts of the Nationwide Inpatient Sample. This is the largest publicly available all payer inpatient database in the United States, capturing 20\% of all US hospital discharges. ${ }^{23}$ This database is assembled annually by the Agency for Healthcare Research and Quality and provides information on more than eight million annual admissions from more than 40 states and more than 1000 hospitals. ${ }^{23}$ The Nationwide Inpatient Sample is a stratified sample of hospitals drawn from existing state databases that make their information available to the Healthcare Cost and Utilization Project (HCUP) with data that can be matched to the American Hospital Association annual survey of hospitals. ${ }^{24}$ It also contains a weighting system that allows for population estimates to be calculated. ${ }^{23}$ The discharge weights are calculated within each sampling stratum as a ratio of discharges in the universe to the sample. ${ }^{24}$ Universe discharges are calculated from the total discharges reported in American Hospital Association hospital survey data from non-rehabilitation hospitals. ${ }^{24}$ Multiple prior studies have utilized this database and the weighting system, focusing on subspecialty cohorts. ${ }^{212} 25-30$

\section{Study population}

We included all patients admitted to hospital between 2002 and 2010. Because the National Inpatient Sample does not contain unique patient identifiers we treated each discharge as an independent event, even if it might represent repeated admissions by the same patient. For each hospital stay we recorded covariates at both patient and hospital level.

\section{Outcomes}

The primary outcome of interest was the ever occurrence of a hospital acquired condition; these were identified using international classification of diseases, ninth edition, clinical modification (ICD-9CM) codes and verified with literature from CMS (see supplementary table). ${ }^{31}$ We tabulated the frequency of individual hospital acquired conditions in this population and the demographic relation to patient and hospital variables. Secondary outcomes of interest included inpatient charges ( $\geq 90$ th centile, $\geq \$ 118505.27$ ) and prolonged length of stay ( $\geq 90$ th centile, $\geq 11$ days).

\section{Covariates}

For the analysis we used nominal covariates such as race (white American, black American, Hispanic, Asian Pacific Islander, Native American, other), payer information (Medicare, Medicaid, private insurance, no charge, self pay, other), and sex (male, female), and ordinal covariates, such as age categories $(<18,19-30$, $31-40,41-50,50-65,66-80,>80$ years) and length of stay. Similarly, hospital factors, such as bed size (small $<200$, medium 201-400, large $>400$ beds), teaching status (teaching, non-teaching), hospital region (north east, Midwest, south, west), and location (rural, urban) were included covariates. We coded missing data fields as missing values and included these in analysis.

The severity of the cause for hospital admission was defined by the All Patient Refined Disease Related Group algorithm (3M Health Information Systems, Wallingford, CT), admission type (emergency, urgent, elective, newborn, trauma, other), and admission source (emergency department, another hospital, other health facility, court or law enforcement, routine admissions, including births, and other sources). The All Patient Refined Disease Related Groups are a system of proprietary risk adjustment methods that builds upon the existing structure of the disease related group and classifies patient admissions in approximately 500 categories with similar clinical features and patterns of resource utilization. The All Patient Refined Disease Related Group from the National Inpatient Sample is categorized into five primary designations: no class specified, minor loss of function, moderate loss of function, major loss of function, and extreme loss of function. To adjust for the severity of injuries requiring admission, we included All Patient Refined Disease Related Group, admission type, and admission source in our models for analysis.

\section{Statistical analysis}

We used survey adjusted univariate and means analysis to calculate population demographics. To assess the relation between the outcome of having at least one hospital acquired condition with patient and hospital factors we conducted multivariable logistic regressions using survey adjusted generalized estimating equations. The primary predictor variable of interest was the timing of admission (weekend versus weekday). We ran secondary analyses to assess the outcome of inpatient mortality with adjustments for the occurrence of a hospital acquired condition and patient, hospital, and severity factors. Similarly, we assessed the impact of neurological comorbidities on occurrence of hospital acquired conditions. We also fit multivariable survey logistic regression models to examine the risk factors of prolonged length of stay and charges. We also conducted a multiple imputation using the methods outlined elsewhere. ${ }^{32}$

For a complex sample data with categorical variables we used a four step procedure for multiple imputation. We created five imputed datasets using the Markov Chain Monte Carlo method and produced a monotone missing data pattern in this large dataset. Using the 


\begin{tabular}{|c|c|c|c|}
\hline \multirow[b]{2}{*}{ Characteristics } & \multicolumn{2}{|l|}{ No (\%) by admission type } & \multirow[b]{2}{*}{$P$ value } \\
\hline & Weekend ( $n=67869246)$ & Weekday $(n=283278574)$ & \\
\hline \multicolumn{4}{|c|}{ No of hospital acquired conditions: } \\
\hline 0 & $64010108(94.3)$ & $272868006(96.3)$ & $<0.01$ \\
\hline$>1$ & $3859138(5.7)$ & $10410568(3.7)$ & $<0.01$ \\
\hline \multicolumn{4}{|l|}{ Inpatient mortality: } \\
\hline Died & $1731783(2.6)$ & 5486665 (1.9) & $<0.01$ \\
\hline Did not die & $66078088(97.4)$ & $277547658(98.0)$ & $<0.01$ \\
\hline Missing & $59365(1.0)$ & $244251(0.1)$ & 0.5037 \\
\hline \multicolumn{4}{|l|}{ Race: } \\
\hline White American & $33408370(49.2)$ & $145479404(51.4)$ & $<0.01$ \\
\hline Black American & $7847842(11.6)$ & $29895824(10.6)$ & $<0.01$ \\
\hline Hispanic & $7219628(10.6)$ & $27677183(9.8)$ & $<0.01$ \\
\hline Asian Pacific Islander & $1469751(2.2)$ & $5568293(2.0)$ & $<0.01$ \\
\hline Native American & $322788(0.5)$ & $1289827(0.5)$ & $<0.01$ \\
\hline Other & $1816947(2.7)$ & $7308392(2.6)$ & $<0.01$ \\
\hline Missing & $15783920(23.3)$ & $66059651(23.3)$ & $<0.01$ \\
\hline \multicolumn{4}{|l|}{ Payer information: } \\
\hline Medicare & 25403449 (37.4) & $104560419(36.9)$ & $<0.01$ \\
\hline Medicaid & $14088122(20.8)$ & $53514983(18.9)$ & $<0.01$ \\
\hline Private insurance & $21708750(32.0)$ & $100817045(35.6)$ & $<0.01$ \\
\hline Self pay & $4153791(6.1)$ & $13406659(4.7)$ & $<0.01$ \\
\hline No charge & $368212(0.5)$ & $1261684(0.4)$ & $<0.01$ \\
\hline Other & 2020948 (3.0) & 9208418 (3.3) & $<0.01$ \\
\hline Missing & $125975(0.2)$ & $509366(0.2)$ & $<0.01$ \\
\hline \multicolumn{4}{|l|}{ Sex: } \\
\hline Female & $28533139(42.0)$ & $116172863(41.0)$ & $<0.01$ \\
\hline Male & $39159898(57.7)$ & $166350325(58.7)$ & $<0.01$ \\
\hline Missing & $176209(0.3)$ & $755387(0.3)$ & $<0.01$ \\
\hline \multicolumn{4}{|l|}{ No of comorbidities: } \\
\hline 0 & $24033497(35.4)$ & $102447854(36.25$ & $<0.01$ \\
\hline 1 & $12241204(18.0)$ & $54782189(19.3)$ & $<0.01$ \\
\hline$\geq 2$ & $30845040(45.4)$ & $122931679(43.4)$ & $<0.01$ \\
\hline Missing & $749506(1.1)$ & $3116851(1.1)$ & $<0.01$ \\
\hline \multicolumn{4}{|l|}{ Age group (years): } \\
\hline$<18$ & $12684772(18.7)$ & 48733195 (17.2) & $<0.01$ \\
\hline $19-30$ & $8631399(12.7)$ & $32684847(11.5)$ & $<0.01$ \\
\hline $31-40$ & $6467587(9.5)$ & 27657135 (9.8) & $<0.01$ \\
\hline $41-50$ & $6389447(9.4)$ & $29082664(10.3)$ & $<0.01$ \\
\hline $51-65$ & $10937715(16.1)$ & $51617250(18.2)$ & $<0.01$ \\
\hline $66-80$ & $12909567(19.0)$ & $58095331(20.5)$ & $<0.01$ \\
\hline$>80$ & $9848759(14.5)$ & $35408152(12.5)$ & $<0.01$ \\
\hline \multicolumn{4}{|c|}{ All Patient Refined Disease Related Group - severity: } \\
\hline No class specified & $44743(0.1)$ & $177046(0.1)$ & $<0.01$ \\
\hline Minor loss of function & $24858060(36.6)$ & $113347265(40.0)$ & $<0.01$ \\
\hline Moderate loss of function & $24897962(36.7)$ & $102808742(36.3)$ & $<0.01$ \\
\hline Major loss of function & $13415105(19.8)$ & $50876315(18.0)$ & $<0.01$ \\
\hline Extreme loss of function & $3903870(5.8)$ & $12952355(4.6)$ & $<0.01$ \\
\hline Missing & $749506(1.1)$ & $3116851(1.1)$ & 0.0693 \\
\hline \multicolumn{4}{|l|}{ Hospital region: } \\
\hline North east & $13434138(19.8)$ & $55559783(19.6)$ & $<0.01$ \\
\hline Midwest & $15550498(22.9)$ & $65236854(23.0)$ & $<0.01$ \\
\hline South & $25455415(37.5)$ & $108789617(38.4)$ & $<0.01$ \\
\hline West & $13429195(19.8)$ & $53692320(19.0)$ & $<0.01$ \\
\hline \multicolumn{4}{|l|}{ Hospital location: } \\
\hline Rural & $9169797(13.5)$ & $37241421(13.1)$ & $<0.01$ \\
\hline Urban & $58431027(86.1)$ & $244913991(86.5)$ & $<0.01$ \\
\hline Missing & $268422(0.4)$ & $1123162(0.4)$ & 0.7530 \\
\hline \multicolumn{4}{|l|}{ Hospital teaching status: } \\
\hline Non-teaching & $37086724(54.6)$ & 152161037 (53.7) & $<0.01$ \\
\hline Teaching & $30514100(45.0)$ & 129994375 (45.9) & $<0.01$ \\
\hline Missing & $268422(0.4)$ & $1123162(0.4)$ & 0.7530 \\
\hline
\end{tabular}

monotone logistic aspect of the multiple imputation procedure, we imputed the missing data. To estimate associations for each imputed dataset we used PROC SURVEYLOGISTIC.

Finally, we synthesized the results from the five surveylogistic models by fully incorporating the variance adjustment from both surveylogistic and multiple imputations (PROC MIANALYZE). ${ }^{32}$ We defined statistical significance as the a level of 0.05. All analyses were performed using SAS 9.3 (Cary, NC).

\section{Results}

From 2002 to 2010, there were 351170803 inpatient admissions, of which 4.1\% (14269706) had at least one hospital acquired condition (table 1). Approximately 81\% (283278574) of inpatient admissions were on a weekday, whereas 19\% (67869246) were on a weekend, and less than 1\% (22983) was missing the day of admission. The frequency of hospital acquired conditions was higher in patients admitted on weekends than on weekdays $(5.7 \% v 3.7 \%)$. Patient and hospital factors differed marginally between populations admitted on weekends and those admitted on weekdays (table 1). Most patients were white (51\%), insured by Medicare (37\%), and male (59\%). Additionally, most weekday admissions were for routine problems (40.1\%), whereas weekend admissions were from the emergency department (44.4\%). In both groups the majority of admissions were for emergencies (table 1). Overall, weekend admissions were associated with more severe problems in terms of admission type, admission source, and All Patient Refined Disease Related Group severity status (table 1).

The most common hospital acquired conditions were falls or trauma (12621763), pressure ulcers (495495), and catheter associated urinary tract infections (432681) (table 2). The frequency of hospital acquired conditions noticeably increased from 2002 to 2010 (1.6\% increase overall) (table 3). Additionally, the most common diagnoses were diabetes mellitus (ICD-9CM: 250.00), hyperlipidemia (272.4), coronary atherosclerosis of a native coronary artery (414.01), outcome of delivery or single liveborn infant (V27.0), and congestive heart failure (428.0). The most common procedures were transfusion of packed cells (99.04), venous catheterization (38.93), manually assisted delivery (73.59), coronary arteriography with two catheters (88.56), and left heart cardiac catheterization (37.22).

\section{Hospital acquired conditions}

In a multivariable logistic regression adjusting for patient (race, payer status, age, sex), hospital (region, location, teaching status, bed size), and severity (All Patient Refined Disease Related Group, admission source, and admission type) factors, the probability of having at least one hospital acquired condition was $25 \%$ higher for patients admitted on weekends compared with weekdays (odds ratio 1.25, 95\% confidence interval 1.24 to $1.26, \mathrm{P}<0.01$ ) (table 4). The day of admission was the strongest predictor for complications, with teaching status $(1.29,1.23$ to $1.35, \mathrm{P}<0.01$, 
Table 1 | Population demographic, 2002-10

\begin{tabular}{|c|c|c|c|}
\hline \multirow[b]{2}{*}{ Characteristics } & \multicolumn{2}{|l|}{ No (\%) by admission type } & \multirow[b]{2}{*}{$P$ value } \\
\hline & Weekend ( $\mathrm{n}=67869$ 246) & Weekday $(n=283278$ 574) & \\
\hline \multicolumn{4}{|l|}{ Hospital bed size: } \\
\hline Small $(<200)$ & 8116805 (12.0) & $34517847(12.2)$ & $<0.01$ \\
\hline Medium (201-400) & $16997083(25.0)$ & 69576844 (24.6) & $<0.01$ \\
\hline Large $(>400)$ & $42486935(62.6)$ & $178060721(62.9)$ & $<0.01$ \\
\hline Missing & $268422(0.4)$ & $1123162(0.4)$ & 0.7530 \\
\hline \multicolumn{4}{|l|}{ Admission type: } \\
\hline Emergency & $36758898(54.2)$ & $104427823(36.9)$ & $<0.01$ \\
\hline Urgent & 10243917 (15.1) & 50133494 (17.7) & $<0.01$ \\
\hline Elective & $6377005(9.4)$ & 71188912 (25.1) & $<0.01$ \\
\hline Newborn & $6399279(9.4)$ & 25783151 (9.1) & $<0.01$ \\
\hline Delivery & $285522(0.4)$ & $599526(0.2)$ & $<0.01$ \\
\hline Other & $24916(0.0)$ & $136489(0.0)$ & $<0.01$ \\
\hline Missing & $7779709(11.5)$ & $31009179(10.9)$ & $<0.01$ \\
\hline \multicolumn{4}{|l|}{ Admission source: } \\
\hline Emergency department & 30113041 (44.4) & 82311348 (29.1) & $<0.01$ \\
\hline Another hospital & 1590294 (2.3) & $7172875(2.5)$ & $<0.01$ \\
\hline Other health facility & $673715(1.0)$ & $3013992(1.1)$ & $<0.01$ \\
\hline Court or law enforcement & $51884(0.1)$ & $229600(0.1)$ & $<0.01$ \\
\hline Routine & $16915596(24.9)$ & $113735341(40.1)$ & $<0.01$ \\
\hline Missing & $18524715(27.3)$ & $76815418(27.1)$ & $<0.01$ \\
\hline
\end{tabular}

teaching versus non-teaching institutions) ranking as another significant predictor (table 4). The associations between medium and large bed size facilities were statistically similar, showing an increased probability of incurring a hospital acquired condition (1.06, $1.01,1.12, \mathrm{P}<0.01$, medium versus small; $1.11,1.06$ to $1.16, \mathrm{P}<0.01$, large versus small). Hospital region, though statistically significant, had marginal associations with the probability of incurring a hospital acquired condition during admission. Patients with comorbid neurological conditions were 35\% more likely than those without to incur a hospital acquired condition during admission $(1.35,1.34,1.37, \mathrm{P}<0.01$; results not shown).

Being from a minority ethnic group and of a younger age were associated with a decreased likelihood of incurring a hospital acquired condition, compared with being older and of white ethnicity (table 4). Patient payer type had mixed associations with the probability of incurring a hospital acquired condition; the likelihood was decreased in Medicare and Medicaid patients, whereas all other payers experienced an increased odds compared with privately insured patients (table 4). Additionally, females were 12\% less likely to incur a hospital acquired condition than male patients in our sample (table 4). More severe All Patient Refined Disease Related Groups, admission types, and admission sources were associated with higher likelihoods of incurring a hospital acquired condition compared with less severe All Patient Refined Disease Related Group, routine admissions, and elective admissions (table 4).

During our multiple imputation analysis, we found that patients admitted on the weekends were $21 \%$ more likely to incur a hospital acquired condition than patients admitted on a weekday $(1.21,1.20$ to 1.22 , $\mathrm{P}<0.01)$. This result was seen to be similar to our non-imputed analysis (see supplementary table).

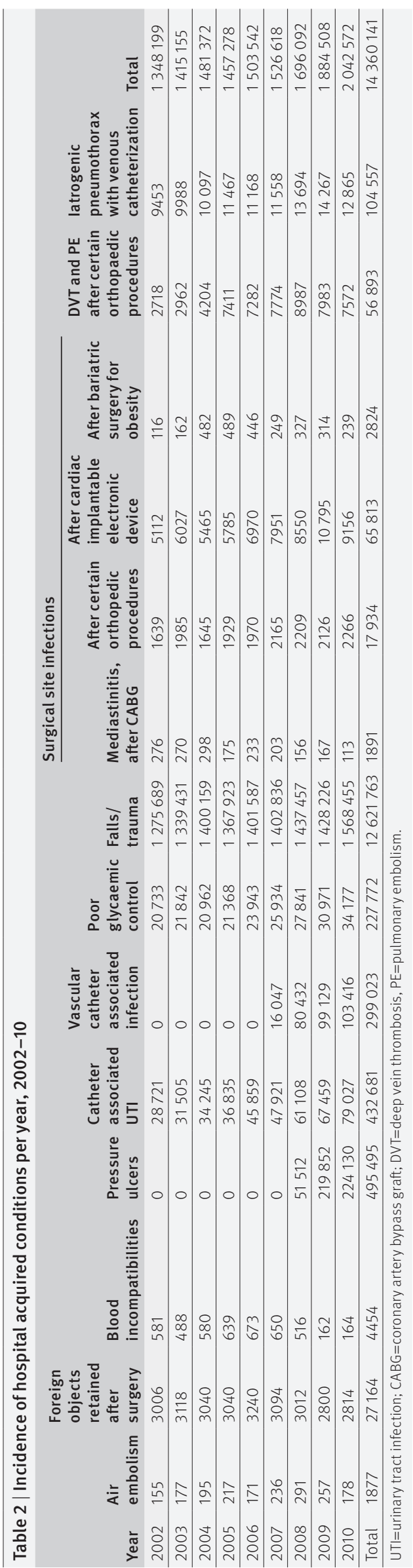




\begin{tabular}{|c|c|c|}
\hline Year & $\begin{array}{l}\text { No with hospital } \\
\text { acquired condition }\end{array}$ & $\%$ of population \\
\hline 2002 & 1345557 & 3.6 \\
\hline 2003 & 1415175 & 3.7 \\
\hline 2004 & 1478298 & 3.8 \\
\hline 2005 & 1453571 & 3.7 \\
\hline 2006 & 1499152 & 3.8 \\
\hline 2007 & 1521342 & 3.8 \\
\hline 2008 & 1683755 & 4.2 \\
\hline 2009 & 1858018 & 4.7 \\
\hline 2010 & 2015318 & 5.2 \\
\hline
\end{tabular}

Higher inpatient charges and prolonged length of hospital stay

The presence of one or more hospital acquired conditions was associated with higher inpatient charges (1.83, 1.77, 1.90, $\mathrm{P}<0.01)$, when adjusting for timing of admission, patient factors (age, race, payer status, sex, admission severity), and hospital factors (region, location, teaching status, bed size). The occurrence of one or more hospital acquired conditions was also associated with prolonged length of stay (1.38, 1.36 to 1.41, $\mathrm{P}<0.01$ ) (table 5). Mean inpatient charges for patients with at least one hospital acquired condition was $\$ 48788$ and length of stay was 6.56 days, significantly greater than patients with no hospital acquired conditions (table 6).

\section{Discussion}

Medical care throughout the world has faced scrutiny and increased public awareness as focus has turned to improving public safety, outcomes, and access. ${ }^{33}$ The current US healthcare focus on public safety and cost effectiveness mandates increased attention to quality outcome measures. Estimates suggest that \$ 9.3bn have been attributed annually to the treatment of potentially preventable medical errors, with recent estimates of $\$ 12$ bn saved with a $17 \%$ reduction in hospital acquired conditions since 2010.634 Determining incidence rates and characterizing the external factors associated with such events is critical. The current study is the first to examine the occurrence of Centers for Medicaid and Medicare Services (CMS) defined hospital acquired conditions-hospital events for which resulting costs of treatment are not federally reimbursed-across all inpatient admissions using a large, administrative database. The data provide baseline frequencies and enable subsequent monitoring of trends over time.

\section{National rates for hospital acquired conditions}

Analysis of 351 million US hospital admissions from 2002 to 2010 at least one hospital acquired condition in $4.8 \%$ of hospital admissions (16.7 million inpatient stays). This rate is concordant with previous estimates for hospital acquired conditions reported across the subspecialty literature (range 2.4-15.0\%). ${ }^{2713}$ Falls were the most common event, occurring in 14 million admissions and accounting for $85 \%$ of all hospital acquired conditions.
Hospital acquired conditions occurred more often among patients admitted on weekends (5.7\%) than among those admitted on weekdays (3.7\%). Weekend admission was associated with a $21 \%$ increased likelihood of incurring a hospital acquired condition (odds ratio $1.25,95 \%$ confidence interval 1.24 to $1.26, \mathrm{P}<0$. 01) after adjusting for patient, hospital, and severity of admission characteristics.

\section{Factors potentially affecting rates of hospital acquired conditions}

Multiple factors may impact the association between weekend admission and the occurrence of a hospital acquired condition. Staff volume (physicians, nurses, and ancillary staff) and level of medical expertise are diminished during weekend shifts. ${ }^{143536}$ Reduced staffing may result in more limited attention to each patient at the point of admission. Further, medical staff often provide weekend coverage for one another and may be less familiar with both the acute and the chronic medical conditions of patients requiring hospital admission. Typically, a reduced infrastructure and fewer resources are allocated towards diagnostic testing and operative intervention on weekends. At weekends, delays have been reported in door to balloon time in the setting of acute myocardial infarction, placement of triple lumen catheters in those with acute myeloid leukemia, and performance of endoscopy in patients with hemorrhagic peptic ulcers and esophageal varices..$^{22}$ 37-39 Delays have been noted in patients with metastatic spine tumors receiving surgical intervention on weekends. ${ }^{40}$ These disparities are not restricted to hospitals in the United States. In a retrospective English population cohort study, the authors reported a decrease in quality and safety of stroke care for patients presenting on weekends. ${ }^{41}$

Numerous studies from multiple countries have shown significant increases in mortality (from 3\% to $20 \%$ ) associated with weekend hospital admissions. ${ }^{18-21} 41-43$ This finding was also consistent with our tertiary analysis, indicating that the likelihood of mortality in patients admitted at weekends was $11 \%$ higher than that of patients admitted on weekdays (odds ratio $1.11,95 \%$ confidence interval 1.10 to $1.12, \mathrm{P}<0.01)$. However, the frequency of preventable hospital events may actually be a more accurate reflection of overwhelmed medical systems in the weekend environment. Such events reflect errors in systems and processes. Though mortality rates are heavily influenced by disease severity, medical comorbidities, and goals of care, hospital acquired conditions represent perceived deficiencies in healthcare delivery and are theoretically preventable. Further, deficiencies in weekend care may not always result in mortality but can impact patient care, length of stay, and hospital costs. Studies have found that delays in endoscopy during weekend admissions for patients presenting with hemorrhage from peptic ulcers and esophageal varices do not result in mortality but are associated with significant increases in hospital charges. ${ }^{37}$ 


\begin{tabular}{|c|c|c|}
\hline Predictors & Odds ratio $(95 \% \mathrm{Cl})$ & $P$ value \\
\hline \multicolumn{3}{|l|}{ Patient predictors } \\
\hline \multicolumn{3}{|l|}{ Admission day: } \\
\hline Weekend & 1.25 (1.24 to 1.26$)$ & $<0.01$ \\
\hline Weekday & Reference & \\
\hline \multicolumn{3}{|l|}{ Race: } \\
\hline White American & Reference & \\
\hline Black American & 0.66 (0.63 to 0.68$)$ & $<0.01$ \\
\hline Hispanic & 0.73 (0.69 to 0.77 ) & $<0.01$ \\
\hline Asian Pacific Islander & 0.65 (0.62 to 0.68$)$ & $<0.01$ \\
\hline Native American & 0.93 (0.84 to 1.03$)$ & 0.17 \\
\hline Other & $0.90(0.85$ to 0.95$))$ & $<0.01$ \\
\hline \multicolumn{3}{|l|}{ Payer information: } \\
\hline Medicare & 0.89 (0.86 to 0.91$)$ & $<0.01$ \\
\hline Medicaid & $0.67(0.66$ to 0.70$)$ & $<0.01$ \\
\hline Private insurance & Reference & \\
\hline Self pay & 1.45 (1.38 to 1.51$)$ & $<0.01$ \\
\hline No charge & $1.16(1.03$ to 1.30$)$ & 0.01 \\
\hline Other & 2.07 (1.97 to 2.18 ) & $<0.01$ \\
\hline \multicolumn{3}{|l|}{ Sex: } \\
\hline Female & 0.88 (0.86 to 0.90$)$ & $<0.01$ \\
\hline Male & Reference & \\
\hline \multicolumn{3}{|l|}{ No of comorbidities: } \\
\hline 0 & Reference & \\
\hline 1 & 0.72 (0.71 to 0.74$)$ & $<0.01$ \\
\hline$\geq 2$ & 0.57 (0.56 to 0.59$)$ & $<0.01$ \\
\hline \multicolumn{3}{|l|}{ Age (years): } \\
\hline$<18$ & 0.47 (0.45 to 0.49$)$ & $<0.01$ \\
\hline $19-30$ & 0.41 (0.40 to 0.42$)$ & $<0.01$ \\
\hline $31-40$ & $0.37(0.35$ to 0.38$)$ & $<0.01$ \\
\hline $41-50$ & 0.41 (0.40 to 0.42$)$ & $<0.01$ \\
\hline $51-65$ & 0.41 (0.40 to 0.42$)$ & $<0.01$ \\
\hline $66-80$ & 0.56 (0.56 to 0.57$)$ & $<0.01$ \\
\hline$>80$ & Reference & \\
\hline \multicolumn{3}{|c|}{ All Patient Refined Disease Related Group - severity: } \\
\hline Minor loss of function & Reference & \\
\hline No class specified & $0.34(0.27$ to 0.43$)$ & \\
\hline Moderate loss of function & 1.40 (1.37 to 1.43$)$ & $<0.01$ \\
\hline Major loss of function & $1.77(1.72$ to 1.83$)$ & $<0.01$ \\
\hline Extreme loss of function & 2.60 (2.50 to 2.71$)$ & $<0.01$ \\
\hline \multicolumn{3}{|l|}{ Hospital predictors } \\
\hline \multicolumn{3}{|l|}{ Hospital region: } \\
\hline North east & Reference & \\
\hline Midwest & 1.14 (1.05 to 1.23$)$ & $<0.01$ \\
\hline South & $1.21(1.13$ to 1.30$)$ & $<0.01$ \\
\hline West & $1.25(1.13$ to 1.37$)$ & $<0.01$ \\
\hline \multicolumn{3}{|l|}{ Hospital location: } \\
\hline Rural & Reference & \\
\hline Urban & 0.97 (0.94 to 1.01$)$ & 0.13 \\
\hline \multicolumn{3}{|l|}{ Hospital teaching status: } \\
\hline Non-teaching & Reference & \\
\hline Teaching & $1.29(1.23$ to 1.35$)$ & $<0.01$ \\
\hline \multicolumn{3}{|l|}{ Hospital bed size: } \\
\hline Small $(<200)$ & Reference & \\
\hline Medium (200-400) & 1.06 (1.01 to 1.12$)$ & $<0.01$ \\
\hline Large $(>400)$ & 1.11 (1.06 to 1.16$)$ & $<0.01$ \\
\hline \multicolumn{3}{|l|}{ Admission type: } \\
\hline Emergency & 2.09 (1.97 to 2.22 ) & $<0.01$ \\
\hline Urgent & $1.42(1.35$ to 1.49$)$ & 0.06 \\
\hline Elective & Reference & \\
\hline Newborn & 0.02 (0.02 to 0.02$)$ & $<0.01$ \\
\hline Trauma & $40.39(23.24$ to 70.21$)$ & $<0.01$ \\
\hline Other & 2.51 (1.67 to 3.76$)$ & $<0.01$ \\
\hline
\end{tabular}

\section{Disease severity}

Severity of illness at the time of admission could predispose to adverse events and hospital acquired conditions. ${ }^{44}$ Further, inability to contact or visit a doctor's office may skew the demographics and disease severity of patients who present for emergency weekend hospital admission..$^{13}$ To deal with this potential confounder, we incorporated the All Patient Refined Disease Related Group algorithm, admission source, and admission type, into our multivariable analysis. The All Patient Refined Disease Related Group algorithm attempts to account for diagnosis severity by characterizing functional impairment. Admissions are classified as minor, moderate, major, and extreme loss of function. Not surprisingly, analysis shows that patients admitted with moderate to extreme loss of function were $34 \%$ to $157 \%$ more likely to incur a hospital acquired condition during their hospital admission. Furthermore, neurologic condition has also been significantly linked to decreased outcome, with our results also noting increased hospital acquired conditions among those with neurologic impairments $(\mathrm{P}<0.01)$.

\section{Secondary outcomes}

The occurrence of hospital acquired conditions was associated with a $76 \%$ higher inpatient hospital charge. The mean charge of hospital stay for patients who experienced at least one hospital acquired condition was \$47066, and for patients who had no hospital acquired condition was $\$ 27092$. The rise in cost was independent of patient and hospital factors or day of admission (odds ratio 1.76, 95\% confidence interval 1.69 to 1.82 , $\mathrm{P}<0.001$ ).

That hospital acquired conditions are associated with increased costs is not surprising. Early data reports on surgical complications suggest a total cost of $\$ 58237$ for each nursing related event (fracture from fall) and $\$ 86833$ per infection. The cost of surgery in the absence of an associated event was reported to be $\$ 18284.45$ Though increases in cost associated with hospital acquired conditions seem implicit, the magnitude of the calculated increase is notable. As current policy withholds reimbursement for costs associated with hospital acquired conditions, the financial burden on treating institutions remains substantial. Hospital acquired conditions are also associated with increased length of stay, independent of admission day, as well as hospital and patient characteristics. Mean length of stay is increased from 4.53 to 6.26 days in patients with at least one hospital acquired condition. This is concordant with findings from previous studies. In patients undergoing head and neck surgeries, deep vein thrombosis was associated with significant increases in length of hospital stay. ${ }^{46}$ Surgical site infections and decubitus ulcers were associated with a 5.6 to 10 day increase in hospital stay. ${ }^{8}$ It is notable that increased length of stay in the presence of hospital acquired conditions is independent of admission day. This suggests that the reported length of stay variables are not confounded by patients admitted on weekends waiting longer in the hospital for availability of relevant tests or their primary doctor. It is not clear 
Table 4 | Predictors of hospital acquired conditions by multivariable analysis

\begin{tabular}{lll} 
Predictors & Odds ratio $(95 \% \mathrm{CI})$ & P value \\
Admission source: & & \\
\hline Emergency department & $2.25(2.11$ to 2.40$)$ & $<0.01$ \\
\hline Another hospital & $2.31(2.08$ to 2.55$)$ & $<0.01$ \\
\hline Other health facility & $1.89(1.76$ to 2.04$)$ & $<0.01$ \\
\hline Court or law enforcement & $1.00(0.67$ to 1.49$)$ & 0.99 \\
\hline Routine & Reference & \\
\hline
\end{tabular}

from the current analysis whether hospital acquired conditions contribute to increased hospital costs and prolonged length of stay or if the reverse is true. The analysis simply establishes an association; causative inference is not possible with the data provided. Increased time spent in hospital typically results in higher inpatient costs and certainly may render an individual susceptible to hospital acquired conditions.

\section{Limitations of this study}

The current study is limited by the natural constraints of a large administrative database. ICD-9 coding within an administrative data source is reported to be $80 \%$ accurate. ${ }^{47}$ Coding is performed by a wide variety of staff without strict oversight. Reported data are therefore subject to information bias and local variability. It is unlikely, however, that the weekend and weekday cohorts would be differentially affected. Also, patients admitted on a Sunday night for elective surgery were included as weekend admits, though we adjusted for this population by controlling for elective admission within our multivariable model.

Two of the codes for hospital acquired conditions were not present in the earlier years of the National Inpatient Sample. Pressure ulcers were not coded prior to 2008 and vascular catheter infections were not coded prior to 2007. This prohibits their inclusion in the analysis of hospital acquired conditions in the years with missing data. This could marginally lower the overall reporting frequency of hospital acquired conditions but would unlikely exhibit differential effects on the weekday and weekend rates of hospital acquired conditions. Furthermore, our study sample includes significant het-

\begin{tabular}{ll}
\hline $\begin{array}{l}\text { Table } \mathbf{5} \mid \text { Inpatient charges and length of stay in relation to hospital acquired conditions } \\
\text { Variables }\end{array}$ & P value \\
\begin{tabular}{lll} 
Higher inpatient charges: & & $<0.01$ \\
\hline Hospital acquired condition & $(95 \% \mathrm{Cl})$ & \\
\hline No hospital acquired condition & Reference & $<0.01$ \\
\hline Prolonged length of stay: & & \\
\hline Hospital acquired condition & $1.38(1.36$ to 1.41$)$ & \\
\hline No hospital acquired condition & Reference & \\
\hline
\end{tabular}
\end{tabular}

Table 6 Mean inpatient costs and length of stay by hospital acquired conditions, 2002-10

\begin{tabular}{lll} 
Variables & $\begin{array}{l}\text { Mean }(95 \% \mathrm{Cl}) \\
\text { Hospital acquired condition }\end{array}$ & No hospital acquired condition \\
\hline Inpatient cost (\$) & $48788.00(47119.23$ to 50 457.25) & $27165.00(26501.40$ to 27 829.20) \\
\hline Length of stay (days) & $6.56(6.46$ to 6.67$)$ & 4.53 (4.49 to 4.57) \\
\hline
\end{tabular}

$\$ 1.00$ (£0.67; €0.93). erogeneity and limits the ability in this mixed cohort to make procedure specific or admission specific conclusions. An inherent limitation in the use of the National Inpatient Sample is not being able to distinguish patients with multiple admissions, resulting in an inability to follow subsequent hospital admissions from the original admission of hospital acquired conditions. Because of this, long term implications of hospital acquired conditions may result in prolonged and subsequent hospital stays that cannot be taken into account. Also, patients who sustain a hospital acquired condition may have subsequent repeat hospital admissions where their prior hospital acquired condition may be either documented as a new occurrence or not documented at all, with the former leading to a possibly inflated estimate. Finally, documentation of hospital acquired conditions does not distinguish events that occur after admission from pre-existing conditions. However, most of the hospital acquired conditions studied are single events or conditions highly unlikely to have occurred prior to hospital admission.

\section{Improving future rates of hospital acquired conditions}

The volume of hospital admissions on weekends is unlikely to decline substantially in the foreseeable future. Added resources and efforts of healthcare administrators and providers are needed to tackle the potential shortcomings in quality of patient care during susceptible periods. Hospital acquired conditions reflect deficiencies in systems and processes. The reasons for an increased frequency of "never events" in patients admitted to hospitals over the weekend is likely multifactorial. Staffing problems are a universal concern. Further training and implementation of prescribed protocols may help mitigate the "weekend effect." Studies attribute human factors such as medical errors to systemic inadequacies within a larger context of less stringent adherence to safety. ${ }^{48} 49$ Improved implementation of protocols for patient care may prevent hospital acquired conditions, especially during times of decreased staffing. It is estimated that at least $20 \%$ of hospital acquired infections are preventable with proper training..$^{50}$ Current nationwide efforts to implement technical practices designed to reduce urinary tract infections are underway. ${ }^{51}$ Such programs, providing a standard for the initiation of common practice treatments, whether on weekends or weekdays, may be of large scale benefit.

\section{Conclusion}

Documentation of hospital acquired conditions represents a reproducible metric for quality of patient care, whereas event occurrence is a large financial burden for healthcare providers and institutions. Evaluation of the frequency of hospital acquired conditions across all medical specialties and admission profiles from 2002-10 showed a significant increase in hospital acquired conditions for patients admitted on weekends, with associated increases in hospital costs and length of patient stay. Improved education, staffing, and protocols may reduce this observed effect. 
Contributors: FJA, TW, and SYC provided substantial contributions to the conception and design of the study, data acquisition, interpretation of data, drafting of the manuscript, and final approval of the version to be published, and is in agreement to be accountable for all aspects of the work. AN provided substantial contributions to the conception and design of the study, data acquisition and analysis, interpretation of the data, and drafting of the manuscript, and is in agreement to be accountable for all aspects of the work. MK-T, NS, APA, and WIM provided substantial contributions to the conception and design of the study, interpretation of the data, drafting of the manuscript, and final approval of the version to be published, and is in agreement to be accountable for all aspects of the work. FJA and WJM are the guarantors. Funding: This study received no specific funding.

Competing interests: All authors have completed the ICMJE uniform disclosure form at www.icmje.org/coi_disclosure.pdf and declare: no support from any organisation for the submitted work; no financial relationships with any organisations that might have an interest in the submitted work in the previous three years; no other relationships or activities that could appear to have influenced the submitted work.

Ethical approval: Not required.

Data sharing: No additional data available.

Transparency: The lead author (FJA) affirms that the manuscript is an honest, accurate, and transparent account of the study being reported; that no important aspects of the study have been omitted; and that any discrepancies from the study as planned (and, if relevant, registered) have been explained.

This is an Open Access article distributed in accordance with the Creative Commons Attribution Non Commercial (CC BY-NC 4.0) license, which permits others to distribute, remix, adapt, build upon this work non-commercially, and license their derivative works on different terms, provided the original work is properly cited and the use is non-commercial. See: http://creativecommons.org/licenses/ by-nc/4.0/.

1 Mehtsun WT, Ibrahim AM, Diener-West M, et al. Surgical never events in the United States. Surgery 2013;153:465-72.

2 Joice GA, Deibert CM, Kates M, et al. "Never events": Centers for Medicare and Medicaid Services complications after radical cystectomy. Urology 2013;81:527-32.

3 Rosenthal MB. Nonpayment for performance? Medicare's new reimbursement rule. N Engl I Med 2007;357:1573-5.

4 Milstein A. Ending extra payment for "never events"-stronge incentives for patients' safety. N Engl/ Med 2009;360:2388-90.

5 Pronovost PJ, Goeschel CA, Wachter RM. The wisdom and justice of not paying for "preventable complications". JAMA 2008;299:2197-9.

6 CMS. Efforts to improve patient safety result in 1.3 million fewer patient harms, 50,000 lives saved and $\$ 12$ billion in health spending avoided. 2014. www.hhs.gov/news/press/2014pres/12/20141202a.html.

7 Champaneria MC, Workman AD, Pham AT, et al. Retrospective Analysis of Never Events in Panniculectomy and Abdominoplasty Patients and Their Financial Implications. Annals of plastic surgery 2013.

8 Lee MK, Dodson TB, Karimbux NY, et al. Effect of occurrence of infection-related never events on length of stay and hospital charges in patients undergoing radical neck dissection for head and neck cancer. Oral Surg Oral Med Oral Pathol Oral Radiol 2013;116:147-58.

9 Teufack SG, Campbell P, Jabbour P, et al. Potential financial impact of restriction in "never event" and periprocedural hospital-acquired condition reimbursement at a tertiary neurosurgical center: a single-institution prospective study. J Neurosurg 2010;112:249-56.

10 Morse BC, Boland BN, Blackhurst DW, et al. Analysis of Centers for Medicaid and Medicare Services 'never events' in elderly patients undergoing bowel operations. Am Surg 2010;76:841-5.

11 Rahman M, Smietana J, Hauck E, et al. Size ratio correlates with intracranial aneurysm rupture status: a prospective study. Stroke 2010;41:916-20.

12 Fargen KM, Rahman M, Neal D, et al. Prevalence of patient safety indicators and hospital-acquired conditions in those treated for unruptured cerebral aneurysms: establishing standard performance measures using the Nationwide Inpatient Sample database. I Neurosurg 2013:119:966-73.

13 Allareddy V, Karimbux NY, Dodson TB, et al. Predictors of never events in patients undergoing radical dissection of cervical lymph nodes. Oral Surg Oral Med Oral Pathol Oral Radiol 2013;115:710-6.

14 Angus DC, Shorr AF, White A, et al. Critical care delivery in the United States: distribution of services and compliance with Leapfrog recommendations. Crit Care Med 2006;34:1016-24.

15 Crowley RW, Yeoh HK, Stukenborg GJ, et al. Influence of weekend hospital admission on short-term mortality after intracerebral hemorrhage. Stroke 2009;40:2387-92

16 Saposnik G, Baibergenova A, Bayer N, et al. Weekends: a dangerous time for having a stroke? Stroke 2007;38:1211-5.
17 Aujesky D, Jimenez D, Mor MK, et al. Weekend versus weekday admission and mortality after acute pulmonary embolism. Circulation 2009;119:962-8.

18 Sharp AL, Choi H, Hayward RA. Don't get sick on the weekend: an evaluation of the weekend effect on mortality for patients visiting US EDs. Am J Emerg Med 2013;31:835-7.

19 Bell CM, Redelmeier DA. Mortality among patients admitted to hospitals on weekends as compared with weekdays. N Engl / Med 2001;345:663-8.

20 Barba R, Losa JE, Velasco M, et al. Mortality among adult patients admitted to the hospital on weekends. Eur J Intern Med 2006; 17:322-4

21 Cram P, Hillis SL, Barnett M, et al. Effects of weekend admission and hospital teaching status on in-hospital mortality. Am J Med 2004;117:151-7.

22 Kostis WJ, Demissie K, Marcella SW, et al. Weekend versus weekday admission and mortality from myocardial infarction. N Engl J Med 2007;356:1099-109.

23 Agency for Healthcare Research and Quality. Healthcare Cost and Utilization Project (HCUP) User Support (HCUP-US). 2014. www.ahrq gov/cpi/about/otherwebsites/hcupnet.ahrq.gov/index.html.

24 Houchens R, Elixhauser A. Final report on calculating Nationwide Inpatient Sample (NIS) variances, 2001. HCUP methods series report No 2003-2, 2001. www.hcup-us.ahrq.gov/reports/methods/ CalculatingNISVariances200106092005.pdf.

25 Wen T, He S, Attenello F, et al. The impact of patient age and comorbidities on the occurrence of "never events" in cerebrovascular surgery: an analysis of the Nationwide Inpatient Sample. J Neurosurg 2014;121:580-6.

26 Attenello FJ, Wang K, Wen T, et al. Health disparities in time to aneurysm clipping/coiling among aneurysmal subarachnoid hemorrhage patients: a national study. World Neurosurg 2014;82:1071-6.

27 Wen T, Deibert CM, Siringo FS, et al. Positioning-related complications of minimally invasive radical prostatectomies. J Endourol 2014;28:660-7.

28 Panaich SS, Badheka AO, Chothani A, et al. Results of ventricula septal myectomy and hypertrophic cardiomyopathy (from Nationwide Inpatient Sample [1998-2010]). Am J Cardiol 2014;114:1390-5.

29 Wen T, Attenello F, He S, et al. Racial and socioeconomic disparities in incidence of hospital acquired complications following cerebrovascular procedures. Neurosurgery 2014;75:43-50.

30 Hanks N, Wen G, He S, et al. Expansion of emergency medical service routing for stroke care in the United States from 2000-2010. West J Emerg Med 2014;15:499-503.

31 CMS. Hospital-Acquired Conditions (HAC) in acute Inpatient Prospective Payment System (IPPS) hospitals. 2014. www.cms.gov/ Medicare/Medicare-Fee-for-Service-Payment/HospitalAcqCond/ downloads/HACFactsheet.pdf.

32 SAS. An introduction to multiple imputation of complex sample data using SAS v9. 2. SAS Global Forum Proceedings. SAS Institute, 2010.

33 Kaiman J SD, Anand A, Kingsley JWP, et al. How sick are the world's healthcare systems? Guardian 2014 Oct 29. www.theguardian.com/ society/2014/oct/29/how-sick-are-worlds-healthcare-systems-nhschina-india-us-germany.

34 Zhan C, Miller MR. Excess length of stay, charges, and mortality attributable to medical injuries during hospitalization. JAMA 2003;290:1868-74.

35 Czaplinski C, Diers D. The effect of staff nursing on length of stay and mortality. Med Care 1998;36:1626-38.

36 Blegen MA, Vaughn T. A multisite study of nurse staffing and patient occurrences. Nurs Econ 1998;16:196-203.

37 Shaheen AA, Kaplan GG, Myers RP. Weekend versus weekday admission and mortality from gastrointestinal hemorrhage caused by peptic ulcer disease. Clin Gastroenterol Hepatol 2009;7:303-10.

38 Horst MA, Stuart J, McKinsey N, et al. Process factors affecting door to percutaneous coronary intervention for acute myocardial infarction patients. Am J Med Qual 2012;27:16-20.

39 Bejanyan N, Fu AZ, Lazaryan A, et al. Impact of weekend admissions on quality of care and outcomes in patients with acute myeloid leukemia. Cancer 2010;116:3614-20

40 Dasenbrock HH, Pradilla G, Witham TF, et al. The impact of weekend hospital admission on the timing of intervention and outcomes after surgery for spinal metastases. Neurosurgery 2012;70:586-93.

41 Palmer WL, Bottle A, Davie C, et al. Dying for the weekend: a retrospective cohort study on the association between day of hospital presentation and the quality and safety of stroke care. Arch Neurol 2012;69:1296-302.

42 Turin TC, Kita Y, Rumana N, et al. Incidence, admission and case-fatality of acute myocardial infarction: weekend versus weekday in a Japanese population: 16-year results from Takashima AMI Registry (1988-2003). Eur J Epidemiol 2009;24:93-100.

43 Hoh BL, Chi YY, Waters MF, et al. Effect of weekend compared with weekday stroke admission on thrombolytic use, in-hospital mortality, 
discharge disposition, hospital charges, and length of stay in the Nationwide Inpatient Sample Database, 2002 to 2007. Stroke 2010;41:2323-8

44 Naessens JM, Campbell CR, Shah N, et al. Effect of illness severity and comorbidity on patient safety and adverse events. Am J Med Qual 2012;27:48-57.

45 Brown SB. Data page. Never events in surgery prove costly. Hosp Health Netw 2008;82:72

46 Hennessey P, Semenov YR, Gourin CG. The effect of deep venous thrombosis on short-term outcomes and cost of care after head and neck cancer surgery. Laryngoscope 2012;122:2199-204.

47 Burns EM, Rigby E, Mamidanna R, et al. Systematic review of discharge coding accuracy. I Public Health (Oxf) 2012:34:138-48.

48 Karsh BT, Holden RJ, Alper SJ, et al. A human factors engineering paradigm for patient safety: designing to support the performance of the healthcare professional. Qual Saf Health Care 2006;15 (Suppl 1):i59-65.

49 Gosbee J. Human factors engineering and patient safety. Qual Saf Health Care 2002;11:352-4

50 Harbarth S, Sax H, Gastmeier P. The preventable proportion of nosocomial infections: an overview of published reports. J Hosp Infect 2003:54:258-66; quiz 321.

51 Fakih MG, George C, Edson BS, et al. Implementing a national program to reduce catheter-associated urinary tract infection: a quality improvement collaboration of state hospital associations, academic medical centers, professional societies, and governmental agencies. Infect Control Hosp Epidemiol 2013;34:1048-54. 